Article

\title{
Effect of Sucrose Concentration on Sucrose-Dependent Adhesion and Glucosyltransferase Expression of $S$. mutans in Children with Severe Early-Childhood Caries (S-ECC)
}

\author{
Wei Zhao ${ }^{\dagger}$, Wenqing $\mathrm{Li}^{\dagger}$, Jiacheng Lin, Zhuoyu Chen and Dongsheng Yu * \\ Guanghua School of Stomatology, Guangdong Provincial Key Laboratory of Stomatology, \\ Sun Yat-sen University, Guangzhou 510055, China; E-Mails: zhaowei3@mail.sysu.edu.cn (W.Z.); \\ wendysums@hotmail.com (W.L.); linjiach@mail.sysu.edu.cn (J.L.); \\ chzhuoy@mail2.sysu.edu.cn (Z.C.)
}

$\dagger$ These authors contributed equally to this work.

* Author to whom correspondence should be addressed; E-Mail: yudsh@mail.sysu.edu.cn;

Tel.: +86-20-8386-2553; Fax: +86-20-8382-2807.

Received: 12 July 2014; in revised form: 14 August 2014 / Accepted: 20 August 2014 /

Published: 9 September 2014

\begin{abstract}
Sucrose, extracellular polysaccharide, and glucosyltransferases (GTFs) are key factors in sucrose-dependent adhesion and play important roles in the process of severe early-childhood caries (S-ECC). However, whether sucrose concentration regulates gtf expression, extracellular polysaccharide synthesis, and sucrose-dependent adhesion is related to the different genotypes of $S$. mutans isolated from ECC in children and still needs to be investigated. In this study, 52 strains of $S$. mutans were isolated from children with S-ECC and caries-free (CF) children. Water-insoluble glucan (WIG) synthesis was detected by the anthrone method, adhesion capacity by the turbidimetric method, and expression of gtf by RT-PCR in an in vitro model containing $1 \%-20 \%$ sucrose. The genotypes of $S$. mutans were analyzed by AP-PCR. The results showed that WIG synthesis, adhesion capacity, and gtf expression increased significantly when the sucrose concentration was from $1 \%$ to $10 \%$. WIG synthesis and gtfB as well as gtfC expression of the $1 \%$ and $5 \%$ groups were significantly lower than those of the $10 \%$ and $20 \%$ groups $(p<0.05)$. There were no significant differences between the $10 \%$ and $20 \%$ groups. The fingerprints of $S$. mutans detected from individuals in the S-ECC group exhibited a significant difference in diversity compared with those from CF individuals $(p<0.05)$. Further, the expression of gtfB and gtfC in the S-ECC group was significantly different
\end{abstract}


among the 1 - to 5-genotype groups $(p<0.05)$. It can be concluded that sucrose-dependent adhesion might be related to the diversity of genotypes of $S$. mutans, and the $10 \%$ sucrose level can be seen as a "turning point" and essential factor for the prevention of S-ECC.

Keywords: sucrose; severe early childhood caries; S. mutans; glucosyltransferases; genotype

\section{Introduction}

Early-childhood caries (ECC) is defined as "the presence of one or more decayed (non-cavitated or cavitated lesions), missing (due to caries) or filled tooth surfaces" in any primary tooth in a child 71 months old or younger. In children younger than three years old, any sign of smooth-surface caries is indicative of severe early-childhood caries (S-ECC) (American Academy of Pediatric Dentistry, 2003). $\mathrm{ECC}$ is a serious public health problem in many developing countries and low socio-economic groups in Western industrialized nations. According to the report of epidemiological studies provided by the Chinese Center for Disease Control and Prevention, the prevalence rate of ECC is as high as $66 \%$ in the Chinese mainland. Therefore, assessing, managing, and preventing ECC are important.

ECC is characterized as a carbohydrate-induced infectious bacterial disease that can cause severe damage to primary dentition. Excessive carbohydrate (especially sucrose) uptake and Streptococcus mutans (S. mutans) are the principal pathogenic factors, according to previous etiologic research [1]. Colonization by $S$. mutans at an early age is considered to correlate with higher caries activity during childhood, and the cariogenicity of these organisms is related, in part, to their ability to colonize and accumulate on tooth surfaces in the presence of sucrose.

Sucrose is the most cariogenic carbohydrate because it is acidogenic and, more importantly, can serve as a substrate for extracellular polysaccharide synthesis by glucosyltransferases (GTFs) of S. mutans [2,3]. In situ studies have also confirmed that a higher concentration and frequency of sucrose exposure increase extracellular polysaccharide concentration in the biofilm matrix, lower fasting $\mathrm{pH}$ values, and enhance enamel demineralization as compared with biofilms formed in the absence of sucrose [4-7]. Clinical studies have also suggested that synthesis of extracellular polysaccharide is related to caries activity in children [8,9]. Extracellular polysaccharide synthesis by GTFs is essential for the establishment of a matrix that enhances the coherence of bacterial cells and adherence to tooth surfaces [10]. Therefore, it is evident that sucrose, GTFs, and extracellular polysaccharide are key factors involved in the sucrose-dependent adhesion of $S$. mutans and the development of ECC. However, currently, studies of these virulence factors and the relationships of clinical isolates of $S$. mutans from ECC are rare.

A previous study found that differences in caries experience in S. mutans-infected children with ECC correlated with the differences in the capacities for extracellular polysaccharide synthesis. The results suggested that $S$. mutans strains appear to differ with regard to their GTFs-mediated virulence. There are several factors that can influence gtf gene transcription, such as carbohydrate availability and source [11-15]. Whether sucrose concentration can regulate gtf expression, extracellular polysaccharide synthesis, and sucrose-dependent adhesion and the nature of the relationship of gtf expression and genotypes of $S$. mutans isolated from children with ECC still need to be investigated. 
Thus, the objective of this study was to use an in vitro culture model to investigate the effects of diverse levels of sucrose on the synthesis of water-insoluble glucan (WIG), capacity for adhesion, and gtf expression of $S$. mutans isolated from the dental plaque of children with S-ECC and caries-free (CF) children. Furthermore, the genotypes of $S$. mutans and their relationship with gtf expression were also examined.

\section{Materials and Methods}

\subsection{Study Population}

Sixty-seven children, aged 2.7 to 5 years old, from the kindergarten affiliated with Sun Yat-sen University (39 boys and 28 girls) were recruited for this study. They were divided into two groups, $32 \mathrm{CF}$ children and 35 children with S-ECC whose decayed, missing, and filled tooth surface (dmfs) scores were $9.3 \pm 5.3$. Written informed consent was obtained from all parents and/or caregivers, and the experimental procedures were approved by the Institutional Ethical Committee of the School of Stomatology, Sun Yat-sen University.

\subsection{Sampling}

Pooled samples of dental plaque were taken with sterile dental probes from buccal surfaces of anterior teeth and the first mandibular molar. The samples were immediately placed in sterilized tubes containing PBS with $2 \%$ sodium thioglycollate, stored on ice, and transferred to the laboratory within $2 \mathrm{~h}[16,17]$.

\subsection{S. mutans Isolation and Identification}

For the detection of $S$. mutans, undiluted samples and $10^{-1}-10^{-3}$ dilutions were cultured on Trypticase Yeast-Extract Cysteine Sucrose Bacitracin (TYCSB) (Difco, Lawrence, Kansas, MI, USA) plates supplemented with $20 \%$ sucrose and $0.2 \mathrm{U}$ bacitracin $\mathrm{mL} / \mathrm{L}$. The plates were incubated at $37{ }^{\circ} \mathrm{C}$ for $48 \mathrm{~h}$ in an atmosphere of $10 \% \mathrm{CO}_{2}, 80 \% \mathrm{~N}_{2}$, and $10 \% \mathrm{H}_{2}$. The UA159 strain was used as the positive control. In addition, 118 strains of pure $S$. mutans were obtained after morphological, biochemical, and physiological identification. All isolates were subjected to PCR for the identification of $S$. mutans.

\subsection{Assessment of the Cariogenicity of S. mutans}

\subsubsection{Culture Conditions}

Purified $S$. mutans isolates were cultured in tryptone-soy base medium with 1, 5, 10, and 20\% sucrose incubated at $37^{\circ} \mathrm{C}$ for $18 \mathrm{~h}$ in the previously described anaerobic atmosphere. (The different sucrose concentrations were chosen according to previous studies conducted by J.A. Cury [4-7]. 


\subsubsection{Adherence Analysis}

The sucrose-dependent adherence of $S$. mutans was determined turbidimetrically as follows $[18,19]$. Purified S. mutans was cultured at an angle of $30^{\circ}$. After incubation, culture tubes were vigorously mixed in a vortex mixer for $5 \mathrm{~s}$, and non-adhering cells were transferred to fresh tubes. Aliquots of $3 \mathrm{~mL}$ of potassium phosphate buffer $(0.05 \mathrm{M}, \mathrm{pH} 7.0)$ were added to the first tube and agitated for $5 \mathrm{~s}$, and then the released cells were transferred to a third tube. The second and third tubes were centrifuged for $5 \mathrm{~min}$ at $5000 \mathrm{~g}$, and the pellets were re-suspended in the same buffer. All test tubes of suspended cells were measured by a spectrophotometer at $550 \mathrm{~nm}$. The percentage of adhered cells was calculated by dividing the cell density of adherent cells by the values of total cell density.

\subsubsection{Water-Insoluble Glucan (WIG) Synthesis}

The quantities of WIG were measured by the anthrone method [20,21]. The cultured tubes were centrifuged for $5 \mathrm{~min}$ at $5000 \mathrm{~g}$, and the pellets were re-suspended three times in $5 \mathrm{~mL} 0.4 \mathrm{~mol} / \mathrm{L} \mathrm{NaOH}$. Then, the supernatant was collected and merged. A solution of $1 \mathrm{~mL}$ of supernatant was added to $3 \mathrm{~mL}$ of anthrone reagent. The mixtures were then heated at $95{ }^{\circ} \mathrm{C}$ for $6 \mathrm{~min}$, followed by spectrophotometric measurement at $625 \mathrm{~nm}$. The amount of WIG was calculated according to the standard curve.

\subsection{RNA Extraction and Reverse Transcription}

Total RNA extraction was performed as previously described [22]. The integrity of RNA was assessed by agarose gel electrophoresis, and the purity of RNA (OD260/OD280) was measured.

\subsection{Reverse Transcription and Polymerase Chain-Reaction (RT-PCR)}

The reverse transcription of mRNA was performed according to the manual provided for the reverse transcription kit (Promega, San Luis Obispo, CA, USA). The primers were designed to amplify gtfB and gtfC [gtfB, (forward) 5'-AGAATACTGATTGGCTGCG-3' and (reverse) 5'-AAGCACCTTTACCA TAGCG-3'; gtfC, (forward) 5'-AGCAGATTCAACTGACGACC-3' and (reverse) 5'-ATGCGGAAA TAGTCTGACGC-3']. PCR amplification was performed, and PCR products were analyzed by electrophoresis.

\subsection{Arbitrarily Primed Polymerase Chain-Reaction (AP-PCR) Analysis}

DNA of $S$. mutans was extracted according to the manufacturer's instructions (Promega, San Luis Obispo, CA, USA). AP-PCR fingerprinting for $S$. mutans was performed with the random primer OPA-02 (5'-TGC CGA GCT G-3'), and PCR products were analyzed by electrophoresis.

\subsection{Statistical Analysis}

Data analyses were performed with SPSS 13.0 (SPSS Inc, Chicago, IL, USA). After a normality test was conducted on the variables of WIG, adhesion ratio, and gtf expression in S. mutans isolated from children with S-ECC and CF children, a general linear model was established, and multifactor variance analysis was used to evaluate the effect of sucrose concentration on the sucrose-dependent adhesion of 
S. mutans. Data from different groups of $S$. mutans and different concentrations of sucrose groups were compared by the least-squares-difference statistical method. The significance value was set at 0.05 .

\section{Results}

\subsection{Detection of S. mutans in Children with S-ECC and CF Children}

S. mutans samples were isolated from 32 of the 35 children with S-ECC (92.4\%) and 20 of the $32 \mathrm{CF}$ children $(62.5 \%)$. The results of the chi-square test revealed that the detection frequencies of $S$. mutans in children with S-ECC were significantly higher than those in CF children $(p<0.05)$. In total, 118 strains of $S$. mutans were obtained from both children with S-ECC and CF children (Table 1).

Table 1. Detection frequencies of $S$. mutans in children with S-ECC were significantly higher than those in caries-free $(\mathrm{CF})$ children $(p<0.05)$.

\begin{tabular}{cccccc}
\hline Groups & Number & Number (\%) & $\begin{array}{c}\text { Strains of } \boldsymbol{S} \text {. mutans } \\
\text { Detection Frequency (\%) }\end{array}$ & dmfs & $\boldsymbol{p}$ \\
\hline S-ECC & 35 & $63(53.4)$ & 92.4 & $9.3 \pm 5.3$ & 0.005 \\
CF & 32 & $55(46.6)$ & 62.5 & 0 & \\
Total & 67 & 118 & & & \\
\hline
\end{tabular}

S-ECC, severe early-childhood caries; CF, caries-free; dmfs, decayed, missing, and filled surfaces of primary teeth.

\subsection{Effect of Sucrose Concentration on the Synthesis of WIG}

According to the results shown in Figure 1, the synthesis of WIG increased significantly with the sucrose concentration, increasing from $1 \%$ to $10 \%$. When the sucrose concentration was above $10 \%$, the synthesis of WIG increased slowly. The highest WIG synthesis, found in the $20 \%$ sucrose group, did not differ significantly from that in the $10 \%$ sucrose group but was significantly higher than that in the $1 \%$ and $5 \%$ sucrose groups $(p<0.05)$.

\subsection{Effect of Sucrose Concentration on the Adhesive Ability of S. mutans}

The adhesion ratio of $S$. mutans isolated from both the ECC and caries-free groups was evidently enhanced when the sucrose concentration was from $1 \%$ to $5 \%$. The adhesion ratio at $1 \%$ was significantly lower than that in the $5 \%, 10 \%$, and $20 \%$ sucrose groups $(p<0.001)$. From $5 \%$ sucrose and above, no significant differences were observed at all sucrose concentrations (Figure 2).

\subsection{Effect of Sucrose Concentration on gtf Expression}

The relative mRNA expression levels of both gtfC (Figure 3) and gtfB (Figure 4) were sucrose-concentration-dependent. The highest gtfB and gtfC expressions were found in the $10 \%$ sucrose group. The expression level decreased when the sucrose concentration was above $10 \%$, but there was no significant difference between the $10 \%$ and $20 \%$ sucrose groups. Therefore, $10 \%$ sucrose can be considered the "turning point" (Figure 3). 
Figure 1. WIG synthesis of $S$. mutans from children with ECC and caries-free children with $1 \%, 5 \%, 10 \%$, and $20 \%$ sucrose. White bars and black bars represent the means (with standard deviations) of WIG synthesized by $S$. mutans isolated from children with ECC and caries-free children. * Denotes statistical significance $(p<0.05)$, and $* * *$ Denotes statistical significance $(p<0.001)$.

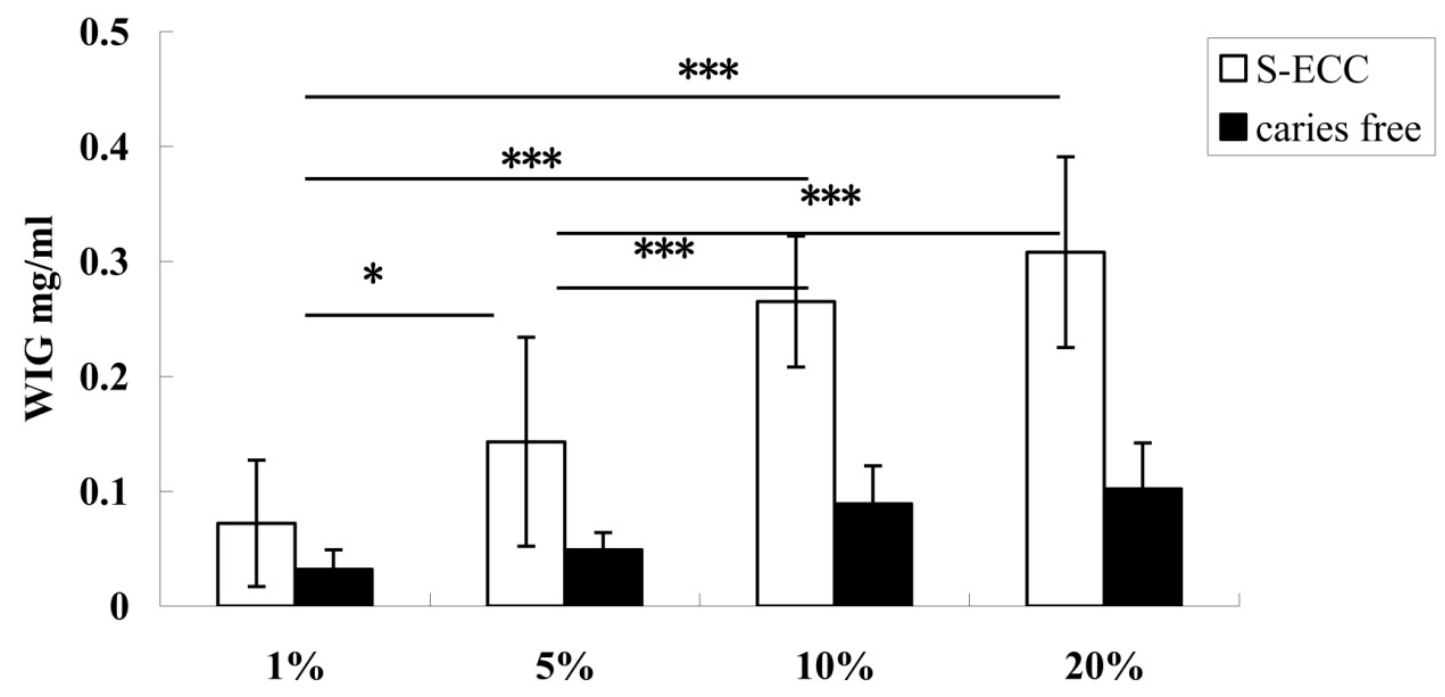

Figure 2. Adhesion ratios of $S$. mutans from children with ECC and caries-free children when the sucrose concentration was $1 \%, 5 \%, 10 \%$, and $20 \%$. The adhesion ratio in the $1 \%$ group was significantly lower than that in the 5\%,10\%, and $20 \%$ groups. There were no significant differences among the 5\%,10\%, and 20\% groups. $* * *$ Denotes statistical significance $(p<0.001)$.

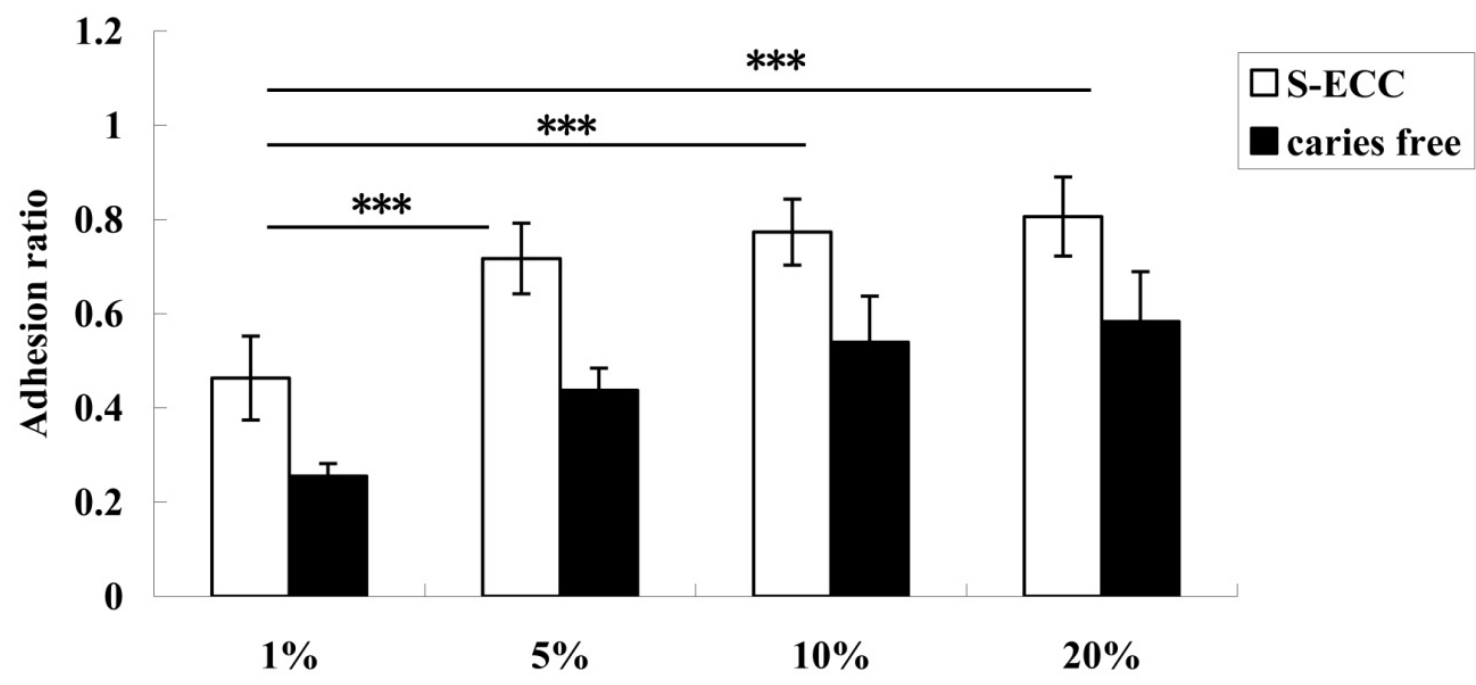


Figure 3. The expression of gtfC in $S$. mutans from children with ECC and caries-free children when the sucrose concentration was $1 \%, 5 \%, 10 \%$, and $20 \%$. The expression level of gtfC increased significantly when the sucrose concentration was $1 \%$ to $10 \%$, and the $1 \%$ group expression was significantly lower than in the $5 \%, 10 \%$, and $20 \%$ groups. $*$ Denotes statistical significance $(p<0.05)$, ** Denotes statistical significance $(p<0.01)$, and $* * *$ Denotes statistical significance $(p<0.001)$.

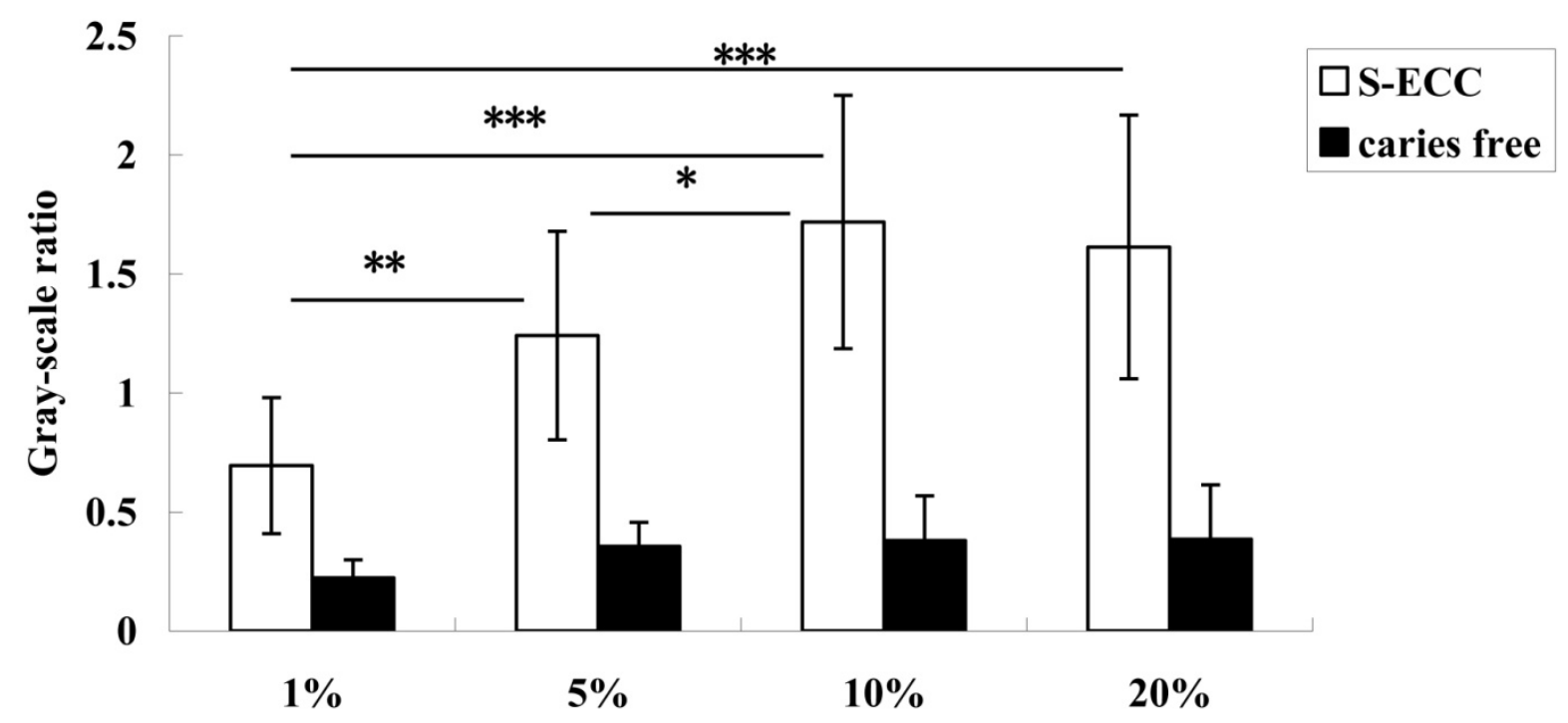

Figure 4. The expression of gtfB in $S$. mutans from children with ECC and caries-free children when sucrose concentration was $1 \%, 5 \%, 10 \%$, and $20 \%$. The expression level of gtfB increased significantly when the sucrose concentration was $1 \%$ to $10 \%$, and the expression in the $1 \%$ and $5 \%$ groups was significantly lower than in the $10 \%$ and $20 \%$ groups. * Denotes statistical significance $(p<0.05)$, ** Denotes statistical significance $(p<0.01)$, and $* * *$ Denotes statistical significance $(p<0.001)$.

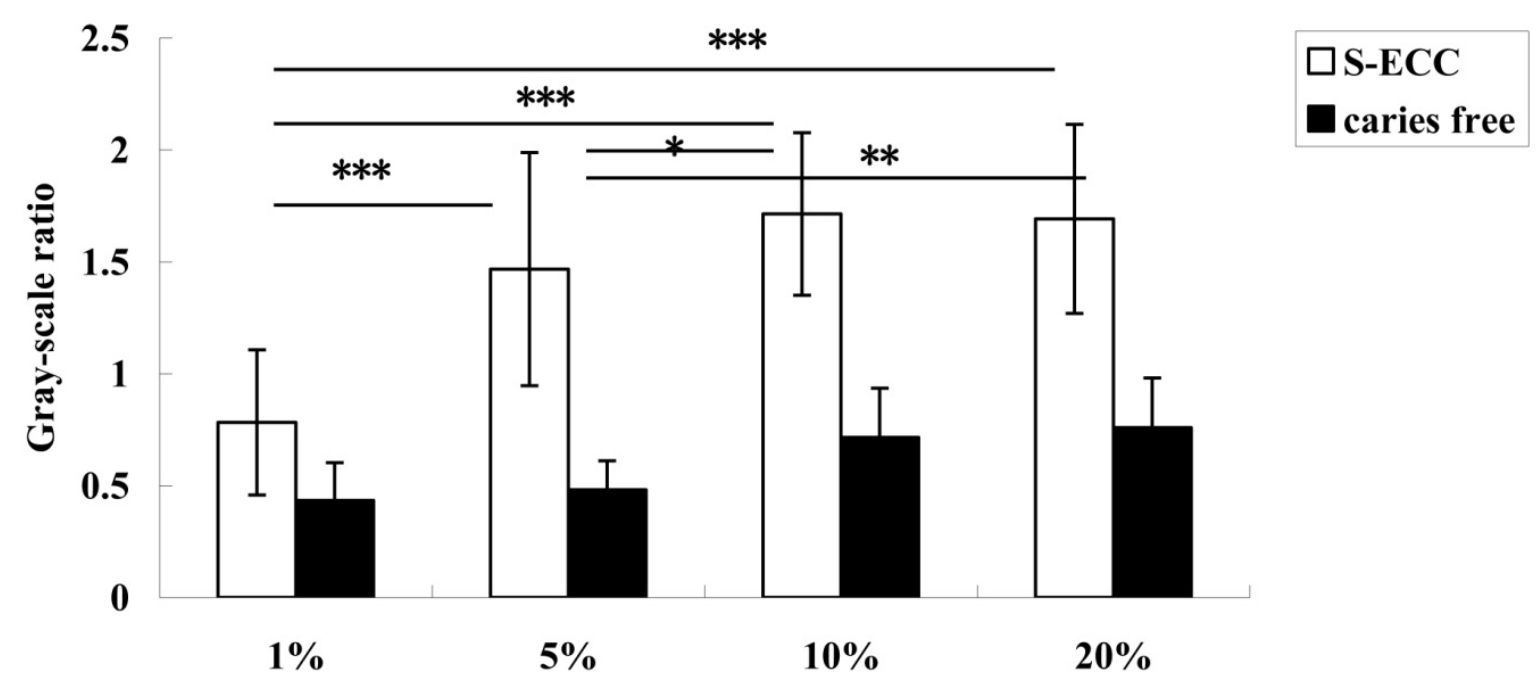




\subsection{The Capacity for Sucrose-Dependent Adhesion of S. mutans from S-ECC and Caries-Free Groups}

To investigate whether different strains of $S$. mutans and different sucrose concentrations interacted during sucrose-dependent adhesion, we compared the capacity for sucrose-dependent adhesion of S. mutans from both S-ECC and CF groups, with strain UA159 used as positive control. As shown in Figure 5, the WIG synthesis, adhesion ratio, and the gene expression levels of gtfB and gtfC were enhanced with increased sucrose concentration and were dose-dependent. This tendency was more obvious in the S-ECC and the UA159 control groups. The capacity for sucrose-dependent adhesion of the strain UA159 detected in this study was higher than that of the strains from the CF group.

Using the general linear model and multifactor variance analysis, we compared the capacity for sucrose-dependent adhesion of $S$. mutans from the S-ECC and caries-free groups. The results indicated that the synthesis of WIG, adhesion ratio, and gene expression of gtfB and gtfC in $S$. mutans isolated from the CF group were significantly lower than those from the S-ECC $(p<0.01)$ group. The results confirmed that the virulence of $S$. mutans from children with S-ECC and caries-free children differed with regard to the synthesis of WIG and gtf expression.

\subsection{Genotypes of S. mutans from the ECC and Caries-Free Groups}

From 1 to 5 genotypes of $S$. mutans were detected in both the S-ECC and CF groups. As shown in Table 2, $87.3 \%$ of children with S-ECC carrying S. mutans harbored more than one genotype, whereas $71 \%$ of the CF children carrying $S$. mutans harbored more than one genotype. Two genotypes of S. mutans accounted for the greatest proportion in the S-ECC and CF groups. However, according to the Mann-Whitney U test (Table 2), the fingerprints of $S$. mutans detected from individuals in the S-ECC group exhibited greater diversity than those from CF individuals $(p<0.01)$. Furthermore, it is interesting that most strains of $S$. mutans in both the S-ECC and CF groups displayed 500-bp and 1000-bp bands, although the genotypes were completely different (Figure 6).

Table 2. The genotypes of $S$. mutans from children with S-ECC and CF children. From 1 to 5 genotypes were detected in both groups.

\begin{tabular}{ccccccc}
\hline \multirow{2}{*}{ Groups } & \multicolumn{7}{c}{ Number (\%) } \\
\cline { 2 - 7 } & 1 Genotype & 2 Genotypes & 3 Genotypes & 4 Genotypes & 5 Genotypes & $\boldsymbol{p}$ \\
\hline S-ECC & $8(12.7)$ & $20(31.7)$ & $17(27)$ & $13(20.6)$ & $5(7.9)$ & 0.003 \\
Caries-free & $16(29)$ & $19(34.5)$ & $12(21.8)$ & $6(10.9)$ & $2(3.6)$ & \\
\hline
\end{tabular}

\subsection{The Relationship of Genotypes and gtf Gene Expression}

To determine whether the gene expression of gtfB and gtfC was related to diverse genotypes of S. mutans, we used multifactor variance analysis, and the results revealed that in the S-ECC group, the expression of gtfB and gtfC in $S$. mutans with 1 to 5 genotypes was significantly different $(p<0.05)$. However, in the CF group, the expression of gtfC was significantly different only among the 1- to 5 -genotype groups $(F=51.971, p<0.05)$. There were no significant differences in gtfB expression among the diverse-genotype groups $(F=0.342, p=0.847)$. 
Figure 5. The capacity for sucrose-dependent adhesion of S. mutans from S-ECC and caries-free groups, with strain UA159 as control. The increase in WIG synthesis (A); adhesion ratio (B); and the expression of gtfC $(\mathbf{C})$ and gtfB (D) was sucrose-concentration-dependent, especially the strains from the S-ECC and UA159 groups.

A

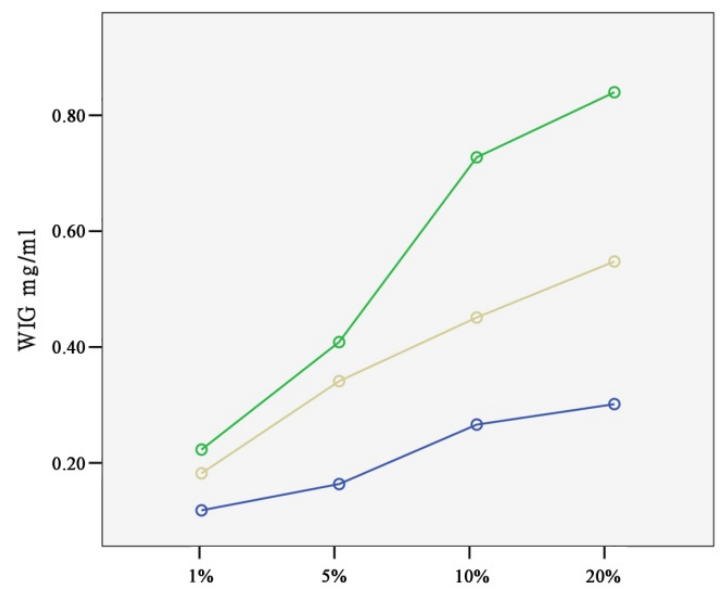

C

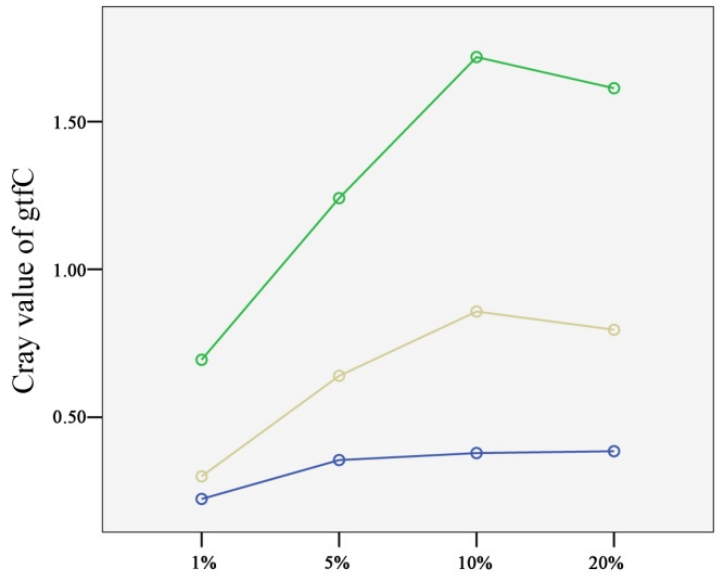

B

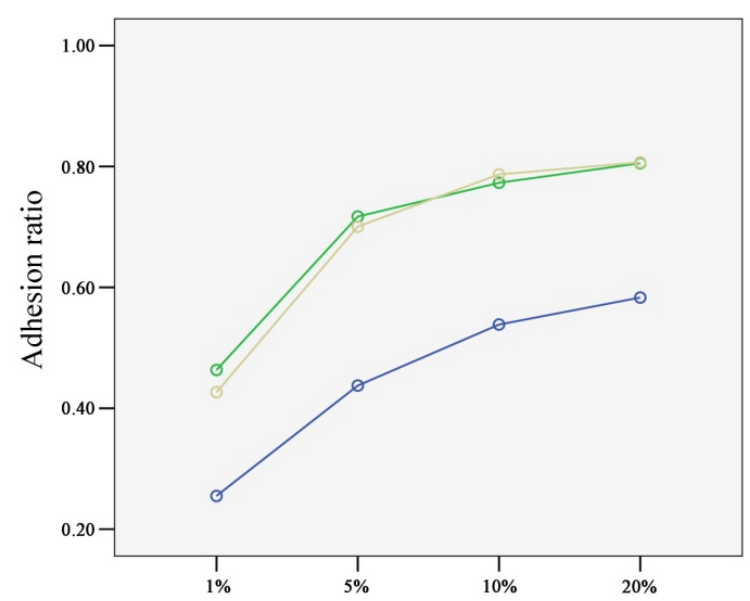

D

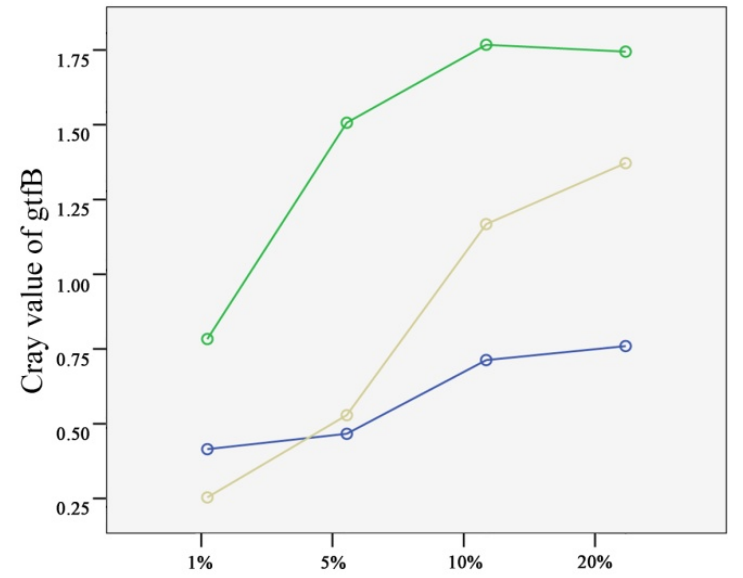


Figure 6. The genotypes of $S$. mutans from children with S-ECC (A) and caries-free (B) children. From 1 to 5 genotypes were detected in the ECC and caries-free groups, but the genotypes differed between the two groups.
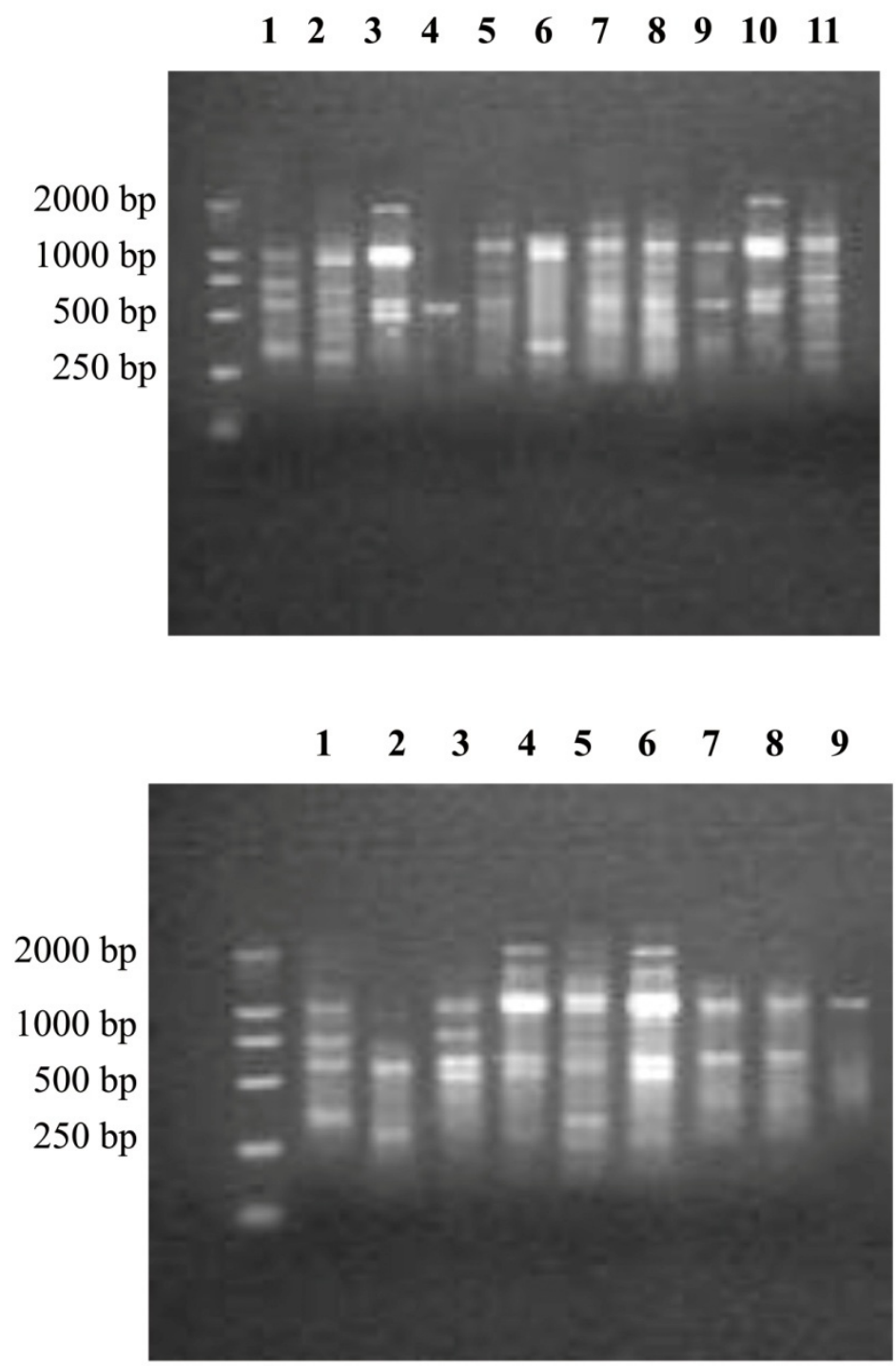

\section{Discussion}

S. mutans is one of the most important pathogenic factors of ECC [23]. The cariogenicity of $S$. mutans is associated with their ability to colonize and accumulate on tooth surfaces, and the formation of sucrose-dependent adhesion plays a key role in the process. Water-insoluble glucans (WIG) synthesized by gtf constitute the major part of the matrix of sucrose-dependent biofilm and provide binding sites for S. mutans for colonization and adherence. Therefore, WIG and gtf are considered the virulence factors of S. mutans. Previous studies have demonstrated that the differences in the capacities for WIG synthesis by $S$. mutans correlate with the caries experience of the children [8]. Thus, S. mutans strains appear to differ with regard to their gtf-mediated virulence. In the current study, we compared WIG synthesis, adhesion ability, and gtf gene expression of 63 strains of $S$. mutans from children with S-ECC with those of the other 55 strains from CF children. The results confirmed that the sucrose-dependent adhesion of $S$. mutans from children with ECC was significantly higher than that from caries-free children at the 
same sucrose level. Furthermore, they also proved that the virulence of $S$. mutans isolated from children with ECC is determined by WIG synthesis and gtf expression.

S. mutans produces at least three genetically separate GTFs, each of which synthesizes a structurally distinct glucan from sucrose. gtfB synthesizes primarily $\alpha$-1,3-insoluble glucan. gtfC produces a mixture of $\alpha-1,6$-soluble glucans and insoluble glucans, and gtfD forms predominantly soluble glucans [24-26]. The genes coding for each gtf isozyme were inactivated, and the resulting mutant strains were analyzed for sucrose-dependent adherence in vitro and for cariogenicity in animal models. These studies indicated that $\mathrm{gtfB}$ and gtfC have the highest association with virulence [27,28]. Factors such as carbohydrate (glucose or sucrose) exposure and environmental $\mathrm{pH}$, which control the expression of gtfB and gtfC, may account for differences in virulence among infecting $S$. mutans strains. The results from some studies have demonstrated that sucrose induces the expression of gtfB [12,14,29]. However, other investigators have reported decreased expression of gtfB and gtfC [30].

Sucrose has been proven to be one of the most important virulence factors in the progression of S-ECC. The role of sucrose in dental caries has been investigated in biofilm models [19,31,32], in vitro models, in situ models, and animal models. The in situ model is composed of healthy adult volunteers who wear acrylic intraoral palatal appliances containing dental enamel blocks and add 1\%, 5\%, 10\%, $20 \%$, or $40 \%$ sucrose solution, 8 times a day [4-7]. This method can mimic the environment of the oral cavity. However, it cannot be applied in children's mouths. The research done at the biofilm level can mimic the development and progression of S-ECC. However, many investigators choose the standard strains of $S$. mutans, such as UA159, for in vitro biofilm formation. There are still some difficulties involved in the study of dental biofilm samples collected from children with S-ECC and CF children. Therefore, in this study, we chose the in vitro model.

In this study, the expressions of gtfB and gtfC were up-regulated with increased sucrose concentration. The expression levels of the $10 \%$ and $20 \%$ sucrose groups were significantly higher than those in the $1 \%$ and $5 \%$ groups $(p<0.01)$. Moreover, no difference was found between the $10 \%$ and $20 \%$ sucrose groups, consistent with the results of the effect of sucrose concentration on WIG synthesis. The 10\% sucrose concentration is regarded as the "turning point" because the expressions of gtfB and gtfC declined when the sucrose concentration was above $10 \%$. Shemesh et al., found that the expression of gtfB in TY culture medium containing $40 \%$ sucrose was slightly lower than that in the medium containing $10 \%$ sucrose, whereas the expression of gtfC under $40 \%$ sucrose is higher than $10 \%$. However, they found no significant difference between the two groups [15]. Therefore, the expressions of gtfB and gtfC cannot be enhanced or down-regulated when the sucrose concentration is higher than $10 \%$.

According to the present study, sucrose concentration is a regulator of gtf expression, WIG synthesis, and adhesion ability of $S$. mutans. A series of in situ studies also found that the threshold of sucrose concentration necessary to form a cariogenic biofilm is $5 \%$, while the cariogenic potential of $10 \%$ and $20 \%$ sucrose was the same [4]. In this study, WIG synthesis and gene expression of gtfB and gtfC in the $5 \%$ sucrose group was significantly lower than that in the $10 \%$ and $20 \%$ groups, and there were no significant differences between the $10 \%$ and $20 \%$ groups. One systemic review presented to inform WHO guidelines for caries prevention showed that caries was lower when free-sugar intake was less than $10 \%$ [33]. Although the result was slightly different from those in the in situ studies, it can be concluded that the sucrose concentration exceeding 5\% has more cariogenic potential for both the 
adhesion of $S$. mutans and the formation of cariogenic biofilm. Therefore, reducing the intake of food containing sucrose concentrations above $5 \%$ could effectively prevent ECC.

The expressions of gtfB and gtfC differed greatly between the S-ECC and CF groups. One study found that substitution of sucrose induced down-regulated expression of gtfB, gtf, gbpB, and vicR of the UA159 strain in a biofilm model [32]. However, the factors regulating the expression of gtf are still unclear. A few studies have targeted the virulence traits of different genotypes of $S$. mutans. Alaluusua et al. [34] found that the polysaccharide synthesis ability of $S$. mutans isolates with different genotypes from caries-active children was different from that of CF children, although the difference was not significant. Another study found that there were more $S$. mutans genotypes with the increased ability to synthesize water-insoluble glucans in caries-active individuals [8,35]. DNA fingerprint analysis revealed that the genotypes of $S$. mutans from 3- to 4-year-old children displayed great genetic diversity. From 1 to 5 genotypes of $S$. mutans were detected as colonizing the oral cavities of children with S-ECC and CF children [36]. In this study, the results of arbitrarily primed PCR also found from 1 to 5 genotypes of $S$. mutans from the S-ECC and CF groups. The fingerprints of $S$. mutans detected from the individuals in the S-ECC group exhibited greater diversity than those from the CF individuals. More importantly, the expression of gtfB and gtfC in the S-ECC group was significantly different among the 1- to 5-genotype groups. This has not yet been reported. The diversity of genotypes was related to the gtf expression levels of $S$. mutans, which suggested that the different gtf coding sequences might result in different virulences of $S$. mutans strains. Therefore, sequence analysis of the gtf gene, especially the promoter and the operon, could help explain the regulation mechanisms of gtf expression and even find molecular markers of ECC, which could be used to screen for highly cariogenic strains of S. mutans.

\section{Conclusions}

S-ECC is a serious public health problem, especially in developing countries. Sucrose has been proven to be an important virulence factor in S-ECC. However, the mechanisms regulating the sucrose-dependent adhesion of $S$. mutans are still not clear. In this study, we found that the synthesis of water-insoluble glucan (WIG), capacity for adhesion, and gene gtf expression in S. mutans isolated from S-ECC were sucrose-concentration-dependent and increased dramatically when sucrose concentration was $1 \%-10 \%$. Apart from this, we found that the fingerprints of $S$. mutans in the S-ECC group showed significant differences in diversity compared with those in the CF group. Therefore, it can be concluded that sucrose-dependent adhesion might be related to the diversity of genotypes of $S$. mutans, and that a $10 \%$ sucrose level can be considered a "turning point". The results of this study will be of great help in S-ECC prevention and oral health promotion for children.

\section{Acknowledgments}

This work was supported by the Guangdong Science and Technology Program Fund (No. 2010B050700004, 2011B050400030), the Guangdong Natural Science Fund (No. S2011020003247), and the National Natural Science Fund (No. 81272554). 


\section{Author Contributions}

As corresponding author, Dongsheng $\mathrm{Yu}$ was responsible for the design and guidance of all parts of the study and gave suggestions for the writing of the manuscript. The major part of the study, including sample collection, S. mutans isolation and identification, adherence analysis, WIG synthesis, and RT-PCR, was done by Wei Zhao and Wenqing Li. DNA extraction and AP-PCR were done by Jiacheng Lin. Zhuoyu Chen kindly helped with sample collection in the kindergarten affiliated with Sun Yat-sen University.

\section{Conflicts of Interest}

The authors declare no conflicts of interest.

\section{References}

1. Berkowitz, R.J. Causes, treatment and prevention of early childhood caries: A microbiologic perspective. J. Can. Dent. Assoc. 2003, 69, 304-307.

2. Adshead, V.M.; Parke, J.M.; Chambers, P.J.; Davies, R.M.; Cole, J.A. An in vitro study of the role of sucrose and interactions between oral bacteria in possible mechanisms of dental plaque formation. Arch. Oral Biol. 1983, 28, 723-727.

3. Rolla, G.; Scheie, A.A.; Ciardi, J.E. Role of sucrose in plaque formation. Scand. J. Dent. Res. 1985 , 93, 105-111.

4. Aires, C.P.; Tabchoury, C.P.; Del Bel Cury, A.A.; Koo, H.; Cury, J.A. Effect of sucrose concentration on dental biofilm formed in situ and on enamel demineralization. Caries Res. 2006, 40, 28-32.

5. Ribeiro, C.C.; Tabchoury, C.P.; Del Bel Cury, A.A.; Tenuta, L.M.; Rosalen, P.L.; Cury, J.A. Effect of starch on the cariogenic potential of sucrose. Br. J. Nutr. 2005, 94, 44-50.

6. Cury, J.A.; Rebello, M.A.; Del Bel Cury, A.A. In situ relationship between sucrose exposure and the composition of dental plaque. Caries Res 1997, 31, 356-360.

7. Cury, J.A.; Rebelo, M.A.; Del Bel Cury, A.A.; Derbyshire, M.T.; Tabchoury, C.P. Biochemical composition and cariogenicity of dental plaque formed in the presence of sucrose or glucose and fructose. Caries Res. 2000, 34, 491-497.

8. Mattos-Graner, R.O.; Smith, D.J.; King, W.F.; Mayer, M.P. Water-Insoluble glucan synthesis by mutans streptococcal strains correlates with caries incidence in 12- to 30-month-old children. J. Dent. Res. 2000, 79, 1371-1377.

9. Nobre dos Santos, M.; Melo dos Santos, L.; Francisco, S.B.; Cury, J.A. Relationship among dental plaque composition, daily sugar exposure and caries in the primary dentition. Caries Res. 2002, 36, 347-352.

10. Forssten, S.D.; Bjorklund, M.; Ouwehand, A.C. Streptococcus mutans, caries and simulation models. Nutrients 2010, 2, 290-298.

11. Wexler, D.L.; Hudson, M.C.; Burne, R.A. Streptococcus mutans fructosyltransferase (ftf) and glucosyltransferase (gtfBC) operon fusion strains in continuous culture. Infect. Immun. 1993, 61, 1259-1267. 
12. Li, Y.; Burne, R.A. Regulation of the gtfBC and $\mathrm{ftf}$ genes of Streptococcus mutans in biofilms in response to $\mathrm{pH}$ and carbohydrate. Microbiology 2001, 147, 2841-2848.

13. Chen, P.M.; Chen, J.Y.; Chia, J.S. Differential regulation of Streptococcus mutans gtfBCD genes in response to copper ions. Arch. Microbiol. 2006, 185, 127-135.

14. Shemesh, M.; Tam, A.; Feldman, M.; Steinberg, D. Differential expression profiles of Streptococcus mutans $\mathrm{ftf}$, gtf and vicR genes in the presence of dietary carbohydrates at early and late exponential growth phases. Carbohydr. Res. 2006, 341, 2090-2097.

15. Shemesh, M.; Tam, A.; Steinberg, D. Expression of biofilm-associated genes of Streptococcus mutans in response to glucose and sucrose. J. Med. Microbiol. 2007, 56, 1528-1535.

16. Caufield, P.W.; Saxena, D.; Fitch, D.; Li, Y. Population structure of plasmid-containing strains of Streptococcus mutans, a member of the human indigenous biota. J. Bacteriol. 2007, 189, $1238-1243$.

17. Li, Y.; Ge, Y.; Saxena, D.; Caufield, P.W. Genetic profiling of the oral microbiota associated with severe early-childhood caries. J. Clin. Microbiol. 2007, 45, 81-87.

18. Hamada, S.; Torii, M. Effect of sucrose in culture media on the location of glucosyltransferase of Streptococcus mutans and cell adherence to glass surfaces. Infect. Immun. 1978, 20, 592-599.

19. Ma, R.; Sun, M.; Wang, S.; Kang, Q.; Huang, L.; Li, T.; Xia, W.W. Effect of high-fructose corn syrup on the acidogenicity, adherence and biofilm formation of Streptococcus mutans. Aust. Dent. J. 2013, 58, 213-218.

20. Munro, C.; Michalek, S.M.; Macrina, F.L. Cariogenicity of Streptococcus mutans V403 glucosyltransferase and fructosyltransferase mutants constructed by allelic exchange. Infect. Immun. 1991, 59, 2316-2323.

21. Van Handel, E. Determination of fructose and fructose-yielding carbohydrates with cold anthrone. Anal. Biochem. 1967, 19, 193-194.

22. Tam, A.; Shemesh, M.; Wormser, U.; Sintov, A.; Steinberg, D. Effect of different iodine formulations on the expression and activity of Streptococcus mutans glucosyltransferase and fructosyltransferase in biofilm and planktonic environments. J. Antimicrob. Chemother. 2006, 57, 865-871.

23. Sonka, J.; Franclova, J. Modification of the anthrone method of blood protein determination. Cas Lek Cesk 1952, 91, 303.

24. Aoki, H.; Shiroza, T.; Hayakawa, M.; Sato, S.; Kuramitsu, H.K. Cloning of a Streptococcus mutans glucosyltransferase gene coding for insoluble glucan synthesis. Infect. Immun. 1986, 53, 587-594.

25. Hanada, N.; Kuramitsu, H.K. Isolation and characterization of the Streptococcus mutans gtfC gene, coding for synthesis of both soluble and insoluble glucans. Infect. Immun. 1988, 56, 1999-2005.

26. Hanada, N.; Kuramitsu, H.K. Isolation and characterization of the Streptococcus mutans gtfD gene, coding for primer-dependent soluble glucan synthesis. Infect. Immun. 1989, 57, 2079-2085.

27. Matsumura, M.; Izumi, T.; Matsumoto, M.; Tsuji, M.; Fujiwara, T.; Ooshima, T. The role of glucan-binding proteins in the cariogenicity of Streptococcus mutans. Microbiol. Immunol. 2003, 47, 213-215.

28. Schneider, R.; Travers, A.; Kutateladze, T.; Muskhelishvili, G. A DNA architectural protein couples cellular physiology and DNA topology in Escherichia coli. Mol. Microbiol. 1999, 34, 953-964. 
29. Burne, R.A.; Chen, Y.Y.; Penders, J.E. Analysis of gene expression in Streptococcus mutans in biofilms in vitro. Adv. Dent. Res. 1997, 11, 100-109.

30. Fujiwara, T.; Hoshino, T.; Ooshima, T.; Hamada, S. Differential and quantitative analyses of mRNA expression of glucosyltransferases from Streptococcus mutans MT8148. J. Dent. Res. 2002, 81, 109-113.

31. Giacaman, R.A.; Campos, P.; Munoz-Sandoval, C.; Castro, R.J. Cariogenic potential of commercial sweeteners in an experimental biofilm caries model on enamel. Arch. Oral Biol. 2013, 58, 1116-1122.

32. Durso, S.C.; Vieira, L.M.; Cruz, J.N.; Azevedo, C.S.; Rodrigues, P.H.; Simionato, M.R. Sucrose substitutes affect the cariogenic potential of Streptococcus mutans biofilms. Caries Res. 2014, 48, 214-222.

33. Moynihan, P.J.; Kelly, S.A. Effect on caries of restricting sugars intake: Systematic review to inform WHO guidelines. J. Dent. Res. 2014, 93, 8-18.

34. Alaluusua, S.; Gronroos, L.; Zhu, X.; Saarela, M.; Matto, J.; Asikainen, S.; Fukushima, K. Production of glucosyltransferases by clinical mutans streptococcal isolates as determined by semiquantitative cross-dot assay. Arch. Oral Biol. 1997, 42, 417-422.

35. Napimoga, M.H.; Kamiya, R.U.; Rosa, R.T.; Rosa, E.A.; Hofling, J.F.; Mattos-Graner, R.; Gonçalves, R.B. Genotypic diversity and virulence traits of Streptococcus mutans in caries-free and caries-active individuals. J. Med. Microbiol. 2004, 53, 697-703.

36. Zhou, Q.; Qin, X.; Qin, M.; Ge, L. Genotypic diversity of Streptococcus mutans and Streptococcus sobrinus in 3-4-year-old children with severe caries or without caries. Int. J. Paediatr. Dent. 2011, $21,422-431$.

(C) 2014 by the authors; licensee MDPI, Basel, Switzerland. This article is an open access article distributed under the terms and conditions of the Creative Commons Attribution license (http://creativecommons.org/licenses/by/3.0/). 